Rev. Biol. Neotrop. 6(2):35-43, 2009

\title{
ISTRIBUIÇÃO ESPACIAL EM PEQUENA ESCALA DE UM ANFÍPODE EXÓTICO EM FRAGMENTO FLORESTAL E PLANTIO DE ESPÉCIES NATIVAS
}

\section{Cristiane Matavelli}

Programa de Pós-graduação em Zoologia, Instituto de Biologia, Universidade Estadual Paulista (UNESP), Caixa Postal 510, Botucatu, 18618-000, São Paulo, Brasil; e-mail: cmatavelli@yahoo.com.br

\section{Wesley Augusto Conde Godoy}

Departamento de Entomologia, Fitopatologia e Zoologia Agrícola, Escola Superior de Agricultura "Luiz de Queiroz" (ESALQ), Universidade de São Paulo, Av. Pádua Dias, 11, Caixa Postal 9, Piracicaba, 13418-900, São Paulo, Brasil; e-mail: wacgodoy@esalq.usp.br

\section{Natalia Oliveira Leiner}

Programa de Pós-graduação em Ecologia, Instituto de Biologia, Universidade Estadual de Campinas (Unicamp), Caixa Postal 6109, Campinas, 13084-971, São Paulo, Brasil; e-mail: naleiner@gmail.com

\section{Marcio Uehara-Prado}

Programa de Pós-graduação em Ecologia, Instituto de Biologia, Universidade Estadual de Campinas (Unicamp), Caixa Postal 6109, Campinas, 13084-971, São Paulo, Brasil; e-mail: muprado@yahoo.com

\begin{abstract}
Resumo: Interferências humanas nos ambientes naturais provocam a quebra de barreiras espaciais e competitivas, as quais podem influenciar a distribuição espacial das espécies. Neste trabalho, descrevemos a distribuição espacial de um anfípode exótico, Talitroides topitotum, em duas áreas distintas: um fragmento florestal e um plantio de espécies arbóreas nativas. Também analisamos possíveis variações dessa distribuição em diferentes épocas do ano e verificamos se a cobertura vegetal e a profundidade da serapilheira podem explicar o padrão de distribuição da espécie. Realizamos análises de distribuição de frequência para verificar os padrões de distribuição da espécie e testes de correlação para verificar o efeito das duas variáveis de estrutura do habitat. A análise de distribuição espacial revelou que T. topitotum tem distribuição agregada em ambas as áreas, o que indica que a espécie tem baixa exigência ambiental para seu estabelecimento ou que ambas as áreas encontram-se abaixo de um limiar mínimo de qualidade ambiental. No entanto, mesmo com esta semelhança, a população do fragmento florestal apresentou maior índice de agregação em relação à população do plantio. Corroborando estudos anteriores, houve correlação negativa entre abundância de $T$. topitotum e profundidade da serapilheira no plantio de espécies nativas. Estudos sobre habitats invadidos podem auxiliar a compreender como espécies invasoras ocupam novos ambientes e os que fatores podem influenciar em sua distribuição espacial.
\end{abstract}

Palavras-chave: Amphipoda, espécie invasora, Floresta Atlântica, perturbação antrópica, Talitroides topitotum.

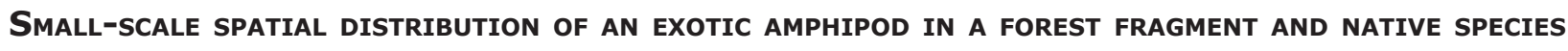
PLANTING

ABstRAct: Anthropogenic interferences in natural environments cause the breakage of spatial and competitive barriers, which may influence the spatial distribution of species. In this study, we describe the spatial distribution of an exotic amphipod, Talitroides topitotum, in two distinct sites, a forest fragment and a plantation of native arboreal species. We analyzed possible variations in this spatial distribution in different periods of the year and verified whether the vegetation cover and the litter 
layer depth may explain the distribution pattern of this species. We performed analyses of frequency distribution to determine the pattern of this species spatial distribution, as well as correlation tests to determine the effect of these two variables of habitat structure. The spatial distribution analysis revealed that $T$. topitotum presents aggregated distribution in both areas, indicating that this species has low environmental demands or that both areas are below a minimum environmental quality threshold. However, even with this similarity, the population in the fragmented site presented a higher index of aggregation when compared with the population of the plantation site. Corroborating previous studies, there was a negative correlation between abundance of $T$. topitotum and litter layer depth in the plantation site. Studies on invaded habitats can help understand how invasive species occupy new environments and the factors that can influence their spatial distribution.

KeY words: Amphipod, invasive species, Atlantic Forest, anthropogenic disturbance, Talitroides topitotum.

\section{INTRODUÇÃO}

introdução de espécies é atual-
mente uma das principais ame-
aças à biodiversidade (Mack et al., 2000; Rodríguez, 2001). O sucesso da invasão de uma espécie depende de sua capacidade em transpor barreiras biogeográficas e de dispersar e manter uma população viável ao longo do tempo (Espínola \& Júlio Junior, 2007).

As invasões podem ocorrer de forma natural ou como resultado de atividades humanas, o que aumenta drasticamente o impacto das invasões em decorrência da alteração e degradação do ambiente (Amin et al., 2009). No entanto, ambas as formas de invasão afetam diretamente as espécies nativas (Fox \& Fox, 1986). Quando essas introduções ocorrem, barreiras geográficas e competitivas são quebradas (Espínola \& Júlio Junior, 2007; Primack \& Rodrigues, 2001), o que afeta tanto as interações entre espécies (como predação, competição e parasitismo) quanto a dinâmica das populações e seus padrões de distribuição espacial (Espínola \& Júlio Junior, 2007; Ricklefs, 2003).

Conhecer o tipo de distribuição espacial de uma espécie é crucial para se compreender a forma como ela utiliza seus recursos (Condit et al., 2000; Ricklefs, 2003). Toda população apresenta um padrão de distribuição espacial diante de um ambiente (Espínola \& Júlio Junior, 2007; Krebs, 1998), o qual pode ser diretamente afetado por perturbações antrópicas, uma vez que alterações como incidência de luz, temperatura, umidade e disponibilidade de recursos modificam todo o ambiente (Costa \& Magnusson, 2003). Apesar do grande número de estudos envolvendo padrões de distribuição espacial com os mais diversos organismos (Condit et al., 2000; Gongalsky et al., 2005; Koivula et al., 1999), são poucos os que relacionam a influência de perturbações antrópicas a esses padrões, tanto em grande quanto em pequena escala.

$\mathrm{O}$ anfípode terrestre Talitroides topitotum (Talitridae) (Burt, 1934), originário de regiões tropicais e subtropicais do Indo-Pacífico (Lemos de Castro, 1972), é atualmente uma espécie cosmopolita (Alvarez et al., 2000). Essa espécie é importante componente da comunidade de invertebrados epigeicos, uma vez que contribui com o processo de decomposição do material vegetal e atua na dinâmica do solo, sendo capaz de aumentar a taxa de oxigenação e diminuir a compactação pelo deslocamento de partículas por sua locomoção (Alvarez et al., 2000; Friend \& Richardson, 1986; Lam \& Ma, 1989). Quando essa espécie é introduzida em novos ambientes, nos quais estão presentes anfípodes terrestres nativos, T. topitotum é capaz de tornar-se a espécie dominante (Alvarez et al., 2000).

T. topitotum foi introduzida no Brasil na década de 1970 (Lemos de Castro, 1972) por intermédio da importação de plantas dos Estados Unidos e da Austrália, principalmente Eucalyptus spp. (Ulian \& Mendes, 1987). Além deste estudo, atualmente há registros de sua distribuição em algumas regiões de Floresta Atlântica, particularmente ao longo da Serra do Mar, nas Regiões Sul e Sudeste do país (Lopes \& Masunari, 2004; Matavelli et al., 2009), nas cidades de São Paulo (Ulian \& Mendes, 1987), Barueri (Lemos de Castro, 1972) e Campinas no estado de São Paulo (observação pessoal), São José dos Pinhais (Lopes \& Masunari, 2004) e Curitiba no estado do Paraná (M. A. 
Bregenski, comunicação pessoal) e Taquara no estado do Rio Grande do Sul (P. B. Araújo, comunicação pessoal).

Os objetivos deste trabalho foram: 1) descrever a distribuição espacial de T. topitotum em duas áreas com diferentes tipos de perturbação - um fragmento florestal e uma área de plantio de espécies arbóreas nativas; 2) analisar possíveis variações na distribuição espacial desses animais em função da época do ano, uma vez que a temperatura influencia sua atividade (Lopes \& Masunari, 2004); e 3) verificar se a cobertura vegetal e a profundidade da serapilheira podem explicar o padrão de distribuição dessa espécie, já que essas variáveis de estrutura do habitat foram relacionadas anteriormente com a abundância de anfípodes terrestres (Kotze \& Lawes, 2008; Spicer \& Tabel, 1996).

Compreender a razão pela qual $T$. topitotum apresenta diferenças quanto à abundância e distribuição espacial em diferentes áreas pode ser considerado o primeiro passo em direção a ações de manejo desta espécie.

\section{Material e métodos}

Realizamos o presente estudo na Fazenda Intermontes ( $\left.24^{\circ} 11^{\prime} 56^{\prime \prime} \mathrm{S}, 48^{\circ} 25^{\prime} 13^{\prime \prime} \mathrm{W}\right)$, localizada no município de Ribeirão Grande, São Paulo, em uma área que compreende 343 ha.
A área de estudo apresenta histórico com vários tipos de interferências antrópicas, como extrativismo seletivo de espécies madeireiras, produção de carvão, além de desmatamento e uso de fogo para o estabelecimento de pastos (Guix, 1994). Atualmente, a área encontra-se como um mosaico de unidades fitofisionômicas, tendo, entre elas, fragmentos de Floresta Atlântica e áreas de reflorestamento, com plantio de espécies arbóreas nativas retiradas desses fragmentos (Nave, 2005).

O clima da região é considerado temperado úmido sem estiagem, de acordo com a classificação de Köppen, tendo temperaturas médias de $18^{\circ} \mathrm{C}$ no mês mais frio e $22^{\circ} \mathrm{C}$ no mês mais quente (Nimer, 1989; Setzer, 1966) e precipitação média de $1.300 \mathrm{~mm}$, variando de $1.100 \mathrm{~mm}$ e $1.500 \mathrm{~mm}$ por ano, com chuvas mais concentradas no verão, principalmente nos meses de dezembro, janeiro e fevereiro (Nimer, 1989; Setzer, 1966). Todas essas características são favoráveis ao estabelecimento de T. topitotum (Alvarez et al., 2000; Ulian \& Mendes, 1987).

A amostragem foi feita em um fragmento florestal e em uma área de plantio de espécies arbóreas nativas. $\mathrm{O}$ fragmento possui uma área de aproximadamente 8 ha, com sub-bosque denso, árvores de grande porte e estratificação bem definida (Tabela 1). A área de plantio, com 6 anos de idade, tem aproximadamente 20 ha e possui espécies como Brachyaria decumbens cv.

Tabela 1 - Espécies arbóreas mais abundantes nas duas áreas utilizadas nesse estudo: o fragmento florestal e o plantio de espécies arbóreas nativas.

\begin{tabular}{lcc}
\hline Espécies arbóreas & Fragmento florestal & Plantio de espécies nativas \\
\hline Acnistus arborescens (L.) Schlecht. & $\mathrm{x}$ & \\
Alchornea triplinervia (Spreng.) Muell. Arg. & $\mathrm{x}$ & $\mathrm{x}$ \\
Baccharis sp. & $\mathrm{x}$ & $\mathrm{x}$ \\
Brachyaria decumbens cv. Basilisk & & $\mathrm{x}$ \\
Croton lindenianus A. Rich. & $\mathrm{x}$ & \\
Prunus myrtifolia (L.) Urb. & $\mathrm{x}$ & $\mathrm{x}$ \\
Senna multifuga (Rich.) Irwin et Barn. & $\mathrm{x}$ & $\mathrm{x}$ \\
Solanum granuloso-leprosum Dunal & $\mathrm{x}$ & \\
Solanum variabile Mart. & $\mathrm{x}$ & \\
Trema micrantha (L.) Blume & $\mathrm{x}$ & \\
Zanthoxylum rhoifolium Lam & &
\end{tabular}

Fonte: Nave (2005). 
Basilisk, que dificultam o desenvolvimento de outras espécies vegetais, pelo abafamento e a ação de substâncias alelopáticas (Nepstad et al., 1998).

Para a amostragem, usamos em cada uma das áreas uma grade de 125 m x 100 m, formada por cinco linhas e cinco colunas, sendo este desenho amostral uma adaptação dos trabalhos de Pinheiro et al. (2002) e Freitas et al. (2002). Cada grade continha 25 armadilhas de queda (pitfall), distantes $25 \mathrm{~m}$ entre si na coluna e $20 \mathrm{~m}$ na linha. As duas áreas são separadas por uma estrada.

Utilizamos como armadilha de queda copos plásticos de $20 \mathrm{~mL}$, enterrados no nível do solo (Pinheiro et al., 2002), contendo álcool 70\% para preservação dos indivíduos capturados e algumas gotas de detergente para quebrar a tensão superficial. Essas armadilhas foram protegidas das chuvas por uma estrutura circular plástica suspensa sobre cada uma delas. As coletas foram realizadas durante quatro dias, mensalmente, entre agosto de 2006 e novembro de 2007, com exceção de dezembro de 2006. Os anfípodes terrestres coletados foram depositados no Museu de Zoologia IB/Unicamp, em Campinas, São Paulo.

Para comparar a distribuição espacial entre as duas áreas, calculamos a abundância média $(x)$, a variância $\left(s^{2}\right)$, o parâmetro de agregação $(k)$ e o qui-quadrado $\left(\chi^{2}\right)$ de cada grade. Segundo Ludwig \& Reynolds (1988), a relação entre a média e a variância do número de indivíduos por armadilha permite avaliar os três tipos básicos de distribuição, que são: $s^{2}=x$ - distribuição aleatória; $s^{2}>x$ - distribuição agregada; e $s^{2}<x$ - distribuição uniforme.

Para verificar o padrão de distribuição espacial de T. topitotum em cada área de estudo, utilizamos as análises de distribuição de frequência, com ajustes aos modelos de distribuição de Poisson e distribuição binomial negativa (Ludwig \& Reynolds, 1988). Entre as características populacionais conhecidas dessa espécie, podemos presumir que sua distribuição espacial seja agregada, já que existe tendência da prole em permanecer perto de seus pais (Alvarez et al., 2000; Lam \& Ma, 1989; Matavelli et al., 2009), sendo este um dos princípios da agregação.
Na distribuição de Poisson, de acordo com Southwood \& Henderson (2006), a probabilidade $P_{x}$ de se encontrar $x$ organismos em uma amostra é dada pela equação:

$$
P_{x}=e^{-m}\left(\frac{m^{x}}{x !}\right)
$$

sendo:

$x=$ número de anfípodes registrados por amostra $(0,1,2 \ldots)$ para uma dada probabilidade $m=$ número médio de anfípodes por amostra $e=$ base dos logaritmos naturais (aproximadamente 2,72)

Na distribuição binomial negativa, conforme Southwood \& Henderson (2006), a probabilidade $P_{x}$ de encontro de $x$ anfípodes em dada amostra é dada pela equação:

$$
P_{x}=\left(1+\frac{m}{k}\right)^{-k} \frac{(k+x-1)}{x !(k-1)}\left(\frac{m}{m+k}\right) x
$$

sendo:

$x=$ número de anfípodes por amostra para o qual a probabilidade é desejada

$m$ = número médio de anfípodes encontrados por amostra

$k$ = parâmetro de agregação

O parâmetro $k$ é um número positivo que reflete o grau de agregação populacional e, quanto mais próximo de zero, maior é a agregação da população (Krebs, 1998). A estimativa mais precisa para o parâmetro $k$ é a da verossimilhança máxima, usada neste estudo. Para ambos os modelos, realizamos os ajustes pelo cálculo das frequências esperadas, que foram confrontadas com as frequências observadas. Para testar a hipótese de igualdade entre as áreas, empregamos o teste de $\chi^{2}$.

Para avaliar possíveis variações na distribuição espacial em função de mudanças nas condições climáticas ocorridas ao longo de um ano, realizamos as análises de distribuição de frequência para os períodos mais favorável (mais quente e úmido, de novembro de 2006 a abril de 2007) e menos favorável (mais frio e seco, de maio de 2007 a outubro de 2007) para T. topitotum (Lam \& Ma, 1989; Lopes \& Masunari, 2004; Matavelli et al., 2009). Com o 
intuito de evitar variações interanuais, os meses de agosto e setembro de 2006 e novembro de 2007 foram excluídos desta análise.

Para verificar a existência de variáveis de estrutura do hábitat que pudessem explicar o padrão de distribuição de T. topitotum, medimos a cobertura vegetal (presença de arbustos menores que $50 \mathrm{~cm}$ e plântulas) e a profundidade da serapilheira (altura da serapilheira sobre o solo) ao lado de cada armadilha. Ambas as variáveis foram citadas em estudos anteriores como potencialmente importantes para o estabelecimento de anfípodes terrestres (Kotze \& Lawes, 2008; Spicer \& Tabel, 1996).

Utilizamos um quadrado de madeira de $50 \mathrm{~cm}$ x $50 \mathrm{~cm}$, dividido em 100 quadrados menores por fios de nylon, para medir a porcentagem de cobertura vegetal (Freitas et al., 2002) e uma régua para medir a profundidade da serapilheira. Realizamos um teste de correlação de Pearson entre essas variáveis e a abundância acumulada de T. topitotum. Para normalizar as variáveis, transformamos os dados de abundância dos anfípodes e a profundidade de serapilheira para raiz quadrada e os dados de cobertura vegetal em arcoseno da raiz quadrada.

\section{Resultados}

Coletamos um total de 116 indivíduos, sendo 60 no fragmento e 56 no plantio. Tanto no fragmento quanto no plantio, o padrão de distribuição espacial foi agregado (Tabela 2 e Figura 1). No entanto, os valores de $k$ indicaram que a população do fragmento apresentou maior grau de agregação $(0,54)$ do que a população do plantio $(1,22)$. Com os dados separados em períodos mais e menos favoráveis para a captura de T. topitotum, o padrão de distribuição também foi agregado.

A abundância de T. topitotum não apresentou correlação significativa com a cobertura vegetal em nenhuma das áreas de amostragem (fragmento $-\mathrm{r}=-0,15, \mathrm{p}=0,46$; plantio $-\mathrm{r}=$ $-0,16, p=0,44)$ e foi negativamente correlacionada com a profundidade da serapilheira somente na área de plantio (fragmento $-r=0,31$, $\mathrm{p}=0,13$; plantio $-\mathrm{r}=-0,55, \mathrm{p}=0,005)$.

\section{Discussão}

T. topitotum apresentou distribuição agregada tanto no fragmento florestal quanto no plantio de espécies nativas. Este tipo de distribuição tem sido relacionado a disponibilidade limitada de refúgios apropriados e a respostas comportamentais à heterogeneidade de habitat, umidade, temperatura, luz, ação do vento, quantidade de serapilheira e pH do solo (Gongalsky et al., 2005; Koivula et al., 1999; Ricklefs, 2003).

Como a abundância e o padrão de distribuição espacial não diferiram entre o fragmento florestal e o plantio de espécies nativas, T. topitotum pode ser considerado um organismo euritópico, ou seja, que apresenta baixa exigência ambiental para seu estabelecimento. Outra hipótese, que não exclui a primeira, é que ambas as áreas estudadas encontram-se em situação de extrema perturbação, abaixo do limiar mínimo de qualidade ambiental e, portanto, igualmente favorável a esta espécie. Estudos feitos na África do Sul demonstraram relação positiva entre a abundância de anfípodes terrestres e o grau de perturbação da área onde estão inseridos (Kotze \& Lawes, 2008).

No entanto, mesmo com essas condições de perturbação, o índice de agregação de $T$. topitotum foi maior para a população do fragmento florestal do que para a população do plantio. Isto pode estar relacionado ao fato de o fragmento florestal apresentar maior heterogeneidade ambiental em relação ao plantio, o que pode beneficiar a formação de alguns locais que sejam mais favoráveis aos anfípodes terrestres do que outros, formando manchas que concentrem mais indivíduos.

Embora alguns estudos tenham demonstrado a existência de correlação entre a abundância de anfípodes terrestres e a profundidade de serapilheira, seus resultados têm sido inconsistentes. Uma correlação positiva entre profundidade de serapilheira e abundância foi encontrada para Arcitalitrus dorrieni (Hunt, 1925) (Talitridae) em Guernsey, no Canal da Mancha (Spicer \& Tabel, 1996). Os autores sugeriram que a maior profundidade da serapilheira propiciaria microclima mais adequado a um animal sensível a dessecação, além do que, maior quantidade de serapilheira resultaria em maior quantidade de cobre, essencial para o metabolismo deste anfípode. Em contraste, foi encontrada correlação negativa para 

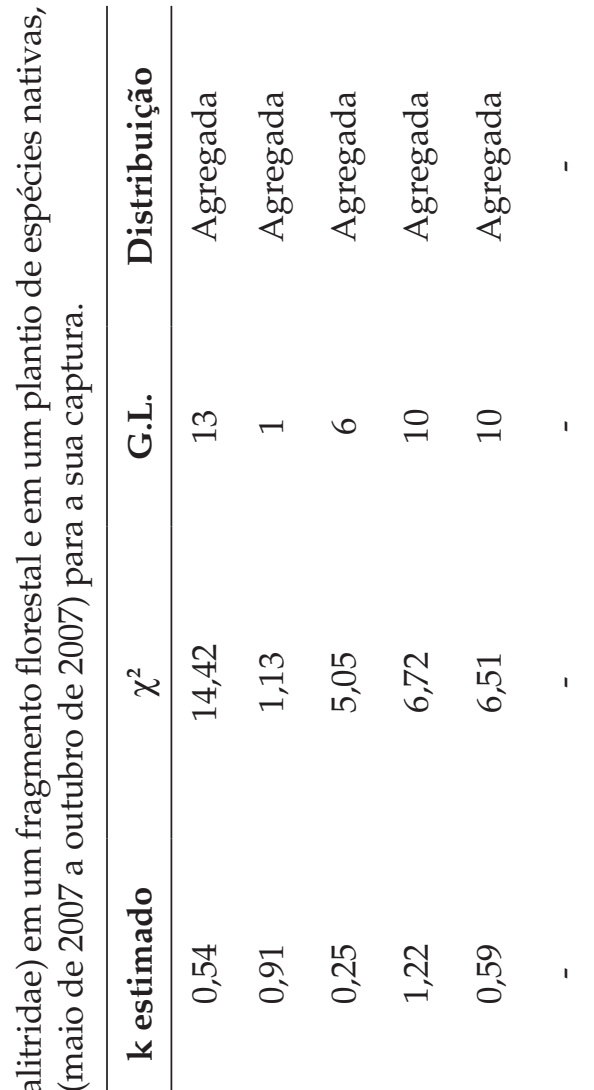


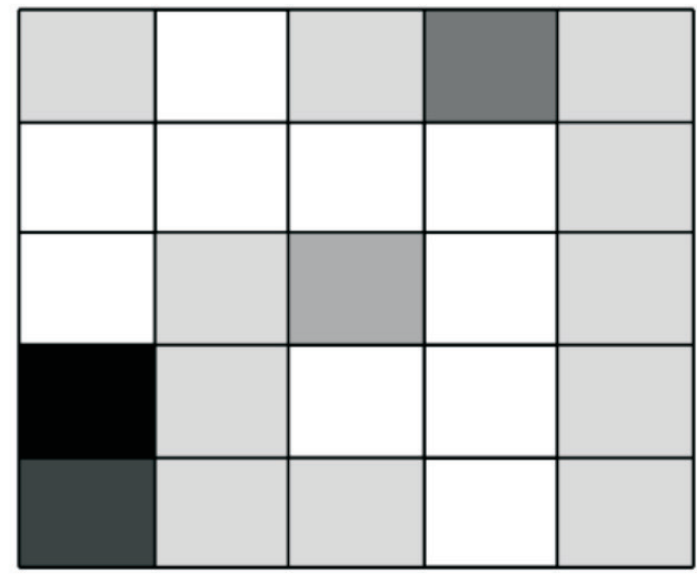

Fragmento florestal

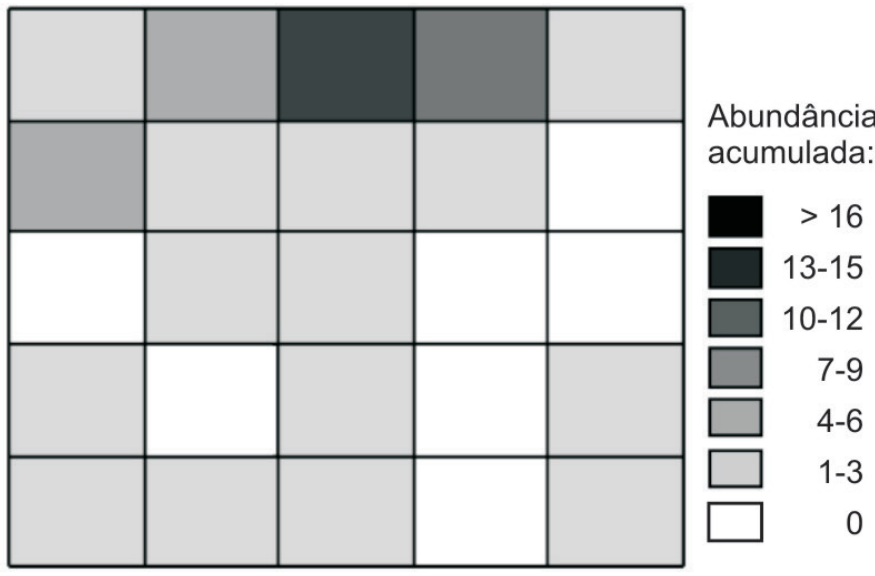

Plantio de espécies arbóreas nativas

Figura 1 - Esquema da distribuição espacial de Talitroides topitotum (Amphipoda, Talitridae) em um fragmento florestal e em uma área de plantio de espécies arbóreas nativas, tendo sido registrada distribuição agregada deste anfípode em ambas as áreas.

Talitriator africana Bate, 1862 (Talitridae) em borda de fragmento em KwaZulu-Natal, na África do Sul (Kotze \& Lawes, 2008).

No presente estudo, encontramos correlação negativa entre abundância de T. topitotum e profundidade de serapilheira somente na área de plantio de espécies nativas, presumivelmente mais perturbada do que o fragmento, o que corrobora o resultado da África do Sul. Kotze \& Lawes (2008) sugeriram algumas hipóteses para esta correlação negativa: 1) quanto mais profunda a serapilheira, maior é a capacidade de sustentar comunidades de predadores epigeicos, que podem suprimir o número de anfípodes terrestres neste ambiente; 2 ) o aumento da densidade de anfípodes terrestres em certas áreas pode elevar a taxa de decomposição da serapilheira desta região, deixando-a mais rasa. Estudos adicionais com T. topitotum são necessários para se testar a consistência do padrão encontrado na Floresta Atlântica.

Nos últimos anos, as modificações ambientais e a translocação de espécies por ação humana têm proporcionado aumento das invasões biológicas em diversos ambientes (Kolar \& Lodge, 2001; Olson, 2006). Este fato tem salientado a importância de se compreender a habilidade de uma espécie exótica em invadir um ecossistema particular (Mack et al., 2000), bem como de se conhecer as características do ambiente que podem favorecer esta espécie (Kühn \& Klotz, 2007). Assim, estudos feitos em habitats invadidos, como este para T. topitotum, podem fornecer subsídios para melhorar a compreensão acerca de como esta espécie invasora ocupa novos ambientes e os fatores que podem influenciar sua distribuição espacial.

Com base nos resultados obtidos neste estudo, podemos concluir que: 1) a abundância de T. topitotum pode ser influenciada pela profundidade da serapilheira; 2) os indivíduos desta espécie são pouco exigentes quanto à seleção de novos locais a serem invadidos; 3) quanto maior for a heterogeneidade dos novos ambientes, provavelmente maior será o grau de agregação desses animais; 4) T. topitotum permanece agregada de forma semelhante ao longo do ano, o que pode facilitar o planejamento do manejo desta espécie, já que não existe a necessidade de aguardar um período do ano mais favorável.

\section{Agradecimentos}

Os autores agradecem à Companhia de Cimento Ribeirão Grande pelo apoio logístico e financeiro durante o período de execução do trabalho, bem como a Odete Lopez Lopes pela identificação de $T$. topitotum. Dois revisores anônimos e o editor M. V. Cianciaruso fizeram comentários que contribuíram substancialmente para a melhoria deste trabalho. Cristiane Matavelli, Marcio Uehara-Prado e Wesley 
Augusto Conde Godoy agradecem ao CNPq pelas bolsas de mestrado (proc. 133010/2008-2), de doutorado (proc. 140116-2004-4) e de produtividade, respectivamente. Natalia Oliveira Leiner agradece à Capes pela bolsa de doutorado.

\section{REFERÊNCIAS}

Alvarez, F., I. Winfield \& S. Cházaro. 2000. Population study of the landhopper Talitroides topitotum (Crustacea: Amphipoda: Talitridae) in central Mexico. J. Nat. Hist. 34: 1619-1624.

Amin, M. N., M. Y. Ali \& M. Salequzzaman. 2009. Identification and impact analysis of invasive species: a case study in the Mongla sea port area of Bagerhat district of Bangladesh. J. Sci. Technol. 4: 35-41.

Condit, R., P. S. Ashton, P. Baker, S. Bunyavejchewin, S. Gunatilleke, N. Gunatilleke, S. P. Hubbell, R. B. Foster, A. Itoh, J. V. LaFrankie, H. S. Lee, E. Losos, N. Manokaran, R. Sukumar \& T. Yamakura. 2000. Spatial patterns in the distribution of tropical tree species. Science 288: 1414-1418.

Costa, F. R. C. \& W. E. Magnusson. 2003. Effects of selective logging on the diversity and abundance of flowering and fruiting understory plants on a Central Amazonian Forest. Biotropica 35: 103-114.

Espínola, L. A. \& H. F. Julio Junior. 2007. Espécies invasoras: conceitos, modelos e atributos. Interciencia 32: 580-585.

Fox, M. D. \& B. J. Fox. 1986. The susceptibility of natural communities to invasion, p. 5766. In: R. H. Groves \& J. J. Burdon (Eds), Ecology of biological invasions. Cambridge, University Press.

Freitas, S. R., R. Cerqueira \& M. V. Vieira. 2002. A device and standard variables to describe microhabitat structure of small mammals based on plant cover. Braz. J. Biol. 62: 795-800.

Friend, J. A. \& A. M. M. Richardson. 1986. Biology of terrestrial amphipods. Annu. Rev. Entomol. 31: 25-48.
Gongalsky, K. B., F. A. Savin, A. D. Pokarzhevskii \& Z. V. Filimonova. 2005. Spatial distribution of isopods in an oak-beech forest. Eur. J. Soil Biol. 41: 117-122.

Guix, J. C. 1994. Intervales, a plenitude da Mata Atlântica, p. 21-35. In: C. Leonel (Ed), Intervales. São Paulo, Fundação para a Conservação e Produção Florestal do Estado de São Paulo, Governo do Estado de São Paulo, Secretaria de Estado do Meio Ambiente.

Koivula, M., P. Punttila, Y. Haila \& J. Niemelä. 1999. Leaf litter and the small-scale distribution of carabid beetles (Coleoptera, Carabidae) in the boreal forest. Ecography 22: 424-435.

Kolar, C. S. \& D. M. Lodge. 2001. Progress in invasion biology: predicting invaders. Trends Ecol. Evol. 16: 199-204.

Kotze, D. J. \& M. J. Lawes. 2008. Environmental indicator potential of the dominant litter decomposer, Talitriator africana (Crustacea, Amphipoda) in Afrotemperate forests. Austral. Ecol. 33: 737-746.

Krebs, C. J. 1998. Ecological methodology. $2^{\text {nd }}$ ed. Benjamin Cummings, New York.

Kühn, I. \& S. Klotz. 2007. From ecosystem invasibility to local, regional and global patterns of invasive species, p. 181-196. In: W. Nentwig (Ed), Biological invasions. Berlin, Springer Berlin Heidelberg. (Ecological Studies, 193).

Lam, P. K. S. \& H. H. T. Ma. 1989. Some observations on the cycle and population dynamics of Talitroides topitotum (Burt) (Amphipoda; Talitridae) in Hong Kong. J. Nat. Hist. 23: 1087-1092.

Lemos de Castro, A. L. 1972. Talitrus (Talitroides) pacificus Hurley, anfípodo terrestre introduzido em São Paulo. Arq. Inst. Biol. S. Paulo, 9: 201-203.

Lopes, O. L. \& S. Masunari. 2004. Distribuição de abundância de Talitroides topitotum (Burt) (Crustacea, Amphipoda, Talitridae) na área de entorno da Usina Hidroelétrica de Guaratuba, Serra do Mar, Guaratuba, Paraná, Brasil. Rev. Bras. Zool. 21: 219-227. 
Ludwig, J. A. \& J. F. Reynolds. 1988. Statistical ecology: a primer on methods and computing. John Wiley \& Sons, New York.

Mack, R. N., D. Simberloff, W. M. Lonsdale, H. Evans, M. Clout \& F. A. Bazzaz. 2000. Biotic invasions: causes, epidemiology, global consequences, and control. Ecol. Appl. 10: 689-710.

Matavelli, C., M. Uehara-Prado, F. P. P. Leite, \& A. V. L. Freitas. 2009. Some aspects of the population ecology of the exotic amphipod, Talitroides topitotum, in an Atlantic Forest Reserve in Brazil. Crustaceana 82: 241-251.

Nave, A. G. 2005. Banco de sementes autóctone e alóctone, resgate de plântulas e plantio de vegetação nativa a Fazenda Intermontes, município de Ribeirão Grande, SP. Tese de doutorado. Escola Superior de Agricultura "Luiz de Queiroz", Universidade de São Paulo, Piracicaba.

Nepstad, D. C., C. Uhl \& C. A. Pereira. 1998. Estudo comparativo do estabelecimento de árvores em pastos abandonados $\mathrm{e}$ florestas adultas da Amazônia Oriental, p. 191-218. In: C. Gascon \& P. Moutinho (Eds), Floresta Amazônica: dinâmica, regeneração e manejo. Manaus, INPA.

Nimer, E. 1989. Climatologia do Brasil. 2 ed. IBGE, Departamento de Recursos Naturais e Estudos Ambientais, Rio de Janeiro.
Olson, L. J. 2006. The economics of terrestrial invasive species: a review of the literature. Agr. Res. Econ. Rev. 35: 178-194.

Pinheiro, F., I. R. Diniz, D. Coelho \& M. P. S. Bandeira. 2002. Seasonal pattern of insect abundance in the Brazilian Cerrado. Austral. Ecol. 27: 132-136.

Primack, B. R. \& E. Rodrigues. 2001. Biologia da conservação. Editora Vida, Londrina.

Ricklefs, R. E. 2003. A economia da natureza. Guanabara Koogan, Rio de Janeiro.

Rodríguez,J.P.2001.Exotic speciesintroductions into South America: an underestimated threat? Biodiv. Conserv. 10: 1983-1996.

Setzer, J. 1966. Atlas climático e ecológico do estado de São Paulo. Comissão Interestadual da Bacia Paraná-Uruguai, Centrais Elétricas de São Paulo, São Paulo.

Southwood, T. R. E. \& P. A. Henderson. 2006. Ecological methods. Blackwell Science, Malden.

Spicer, J. I. \& H. Tabel. 1996. Notes on the occurrence of the introduced landhopper Arcitalitrus dorrieni (Hunt, 1925) on Guernsey, Channel Islands. J. Nat. Hist. 30: 1625-1632.

Ulian, G. B. \& E. G. Mendes. 1987. Preferences of a terrestrial amphipod, Talitrus (Talitroides) pacificus, Hurley, 1955, towards some environmental factors. Rev. Bras. Biol. 47: 247-256.

Recebido em 10.VI.2009 